\title{
Vehicle Automatic Vertical \& Horizontal Parking Trajectory Prediction and Fuzzy Control
}

\author{
Jinqing $\mathrm{Li}^{1, \mathrm{a}}$,Jindong Zhang ${ }^{1,2, \mathrm{~b}, *}$, Weikai Wang ${ }^{1, \mathrm{c}}$, Chengliang Liu ${ }^{1, \mathrm{~d}}$, \\ Hongyan Guo ${ }^{1, e}$ and Kuo Zhang ${ }^{1, f}$
}

${ }^{1}$ College of Computer Science and Technology, Jilin University, Changchun, 130012, China

${ }^{2}$ Key Laboratory of Symbol Computation and Knowledge Engineering of the Ministry of Education, Jilin University, Changchun,130012, China

a1468666205@qq.com, bzhangjindong_100@163.com, c1213786842@qq.com, d996333274@qq.com, e331448369@qq.com, f756436761@qq.com

Keywords: Vertical Parking, Horizontal Parking, Trajectory Prediction, Fuzzy.

\begin{abstract}
Automatic parking system is a non-accurate modeling system, and the fuzzy algorithm is an excellent choice for the non-accurate modeling. Based on the vehicle kinematics modeling and the experience of artificial parking, we establish the horizontal and vertical models of the vehicle automatic trajectory and the control system through fuzzy inference. By fuzzy controller, parking optimal trajectory can control the speed of the vehicle when reversing garage, and at the same time, the fuzzy controller can control the vehicle body posture reverse garage. These two controls work closely together to achieve accurate body trajectory. The accuracy of the controller is verified by the simulation experiment.
\end{abstract}

\section{Introduction}

At present, the automatic parking system applied to mass production cars is only a secondary system, and cannot achieve automatic trajectory. That means, the driver still needs to control the gear, throttle and brakes according to the system prompt. There are two main types of automatic parking system, one is the open loop parking control based on path planning, which controls non-learning trajectory through the controller.Another is learning parking based on fuzzy algorithm and neural network, which parks through the study and simulation of artificial experience. The former's location of real-time is not good, consuming more time to correct the position errors. The latter can reduce positional errors in real time so that it can achieve accurate parking [1].

Because there will be a problem that the speed is not constant in the process of the vehicle's reversing into garage, excessive speed will cause that the required effective parking space is too large to be satisfied and too slow speed will make the parking time increase so that it cannot park into garage in a certain time.This paper adopted two levels of fuzzy controller. The first-level fuzzy controller controlled the vehicle speed, kept the speed of the vehicle in a proper range,and realized the dynamic stability of the system velocity.According to the artificial experience,based on fuzzy algorithm, the second-level fuzzy controller realized the dynamic control of the vehicle's steering angle, correcting astern position and vehicle posture in real time. In the process of parking, there mainly has the following three phases: the first stage, it used ultrasonic sensors to read information around the vehicle, and determined the starting position of the car parking; The second phase, it read the vehicle speed, adjusted the speed and used ultrasonic sensor to collect location information back to the controller, which decided position adjustment; In the third phase, it hit front wheel to complete parking process. 


\section{Kinematic model and path of the vehicle parking Analysis.}

In the process of vehicle steering, when the outer steering wheel reaches the maximum steering angle, it gets minimum turning radius. Under the current parking resource constraints, it reduces the size of vehicle parking space within a reasonable range. To make parking space as small as possible, we should try to reduce the turning radius of the vehicle parking process, in order to minimize the use of effective parking space and achieve the rational use of parking space. Based on the Ackerman Angle, this system established the model to carry on the parking trajectory prediction [2,3]. Assuming the the front wheels of the vehicle steer, the rear wheels and the vehicle were always parallel. $l$ was vehicle length, $w$ was vehicle width, $s$ is wheel base, $b$ is wheel tread, $a$ was front suspension length, $c$ was overhang length, $\alpha$ was steering angle of outside steering wheel, $\beta$ was steering angle of internal steering wheel,all of these had maximum value. O' was the reference center point of study, $R$ was steering radius of vehicle parking trajectory, $R_{0}$ was outside steering wheel turning radius.From the corner of Ackerman Angle knowledge, when $\alpha$ reached its maximum value, $R_{0}$ got the minimum value, that was:

$$
R_{0_{-} \min }=\frac{s}{\sin \alpha_{\max }}
$$

On the basis of this, the minimum radius of the reference center point $O^{\prime}$ of the vehicle was established, expand the following trajectory prediction and calculation, at this time there was a minimum radius of $O^{\prime}$ :

$$
R_{\min }=R_{\alpha_{\max }} \cos \alpha_{\text {max }}=s \cot _{\alpha_{\max }}
$$

Assuming that in the process of the parking origin $\mathrm{O}$ can be met, Fig. 1 showed a schematic diagram of the automatic vertical parking trajectory prediction. The paper used two phases of the parking trajectory for parking, the two arrows in the figure indicated that the trajectory was the vertical parking path, the first phase was preparation for parking. According to different starting position, calculate different origins from the visual parking. The second phase was the real stage of reversing parking. Vehicle parked in the center of parking space eventually. The meaning of the parameters in the diagram was as follows: $W$ was the width of parking space, $H$ was the depth of parking spaces, ss was the distance from the right side of the vehicle to the barriers, $R_{\min }$ was trajectory radius of reverse phase, $R_{1_{-} \text {min }}$ was the trajectory radius of the vehicle to the designated position in the preparation phase. $R_{C 1}$ and $R_{D 1}$ were parking trajectory radius of $C 1, D 1$. In this trajectory, make rear axle center as study reference center point and in the forward phase take the steering wheel to maximum steering angle. From the Ackerman Angle knowledge, the second phase minimum parking radius was:

$$
R_{1_{-} \min }=\sqrt{R_{0 \_ \text {min }}^{2}-s^{2}}-\frac{b}{2}
$$

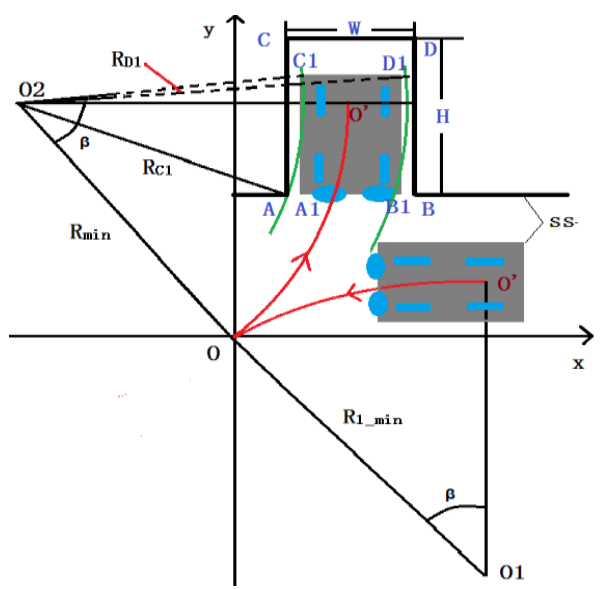

Fig. 1 Schematic diagram of automatic vertical parking path planning 
The minimum parking radius for the first phase was:

$$
R_{\min }=\sqrt{2}\left(s s+\frac{w}{2}+R_{1_{-} \min }\left(1-\frac{\sqrt{2}}{2}\right)+l-c\right)
$$

There may be two collision points in the vertical parking process, they are: the collision between the point of the obstacle of the vehicle $A$ and the side of the vehicle $A 1 C 1$, and the collision between the point of the obstacle of the garage side $B D$ and the side of the vehicle $D 1$. In order to avoid the two collisions, the minimum effective parking space should be set. If it does not meet the minimum size, it will not be allowed to park here.There are two critical conditions to meet the minimum effective parking space. One is that the garage point $A$ and the side of the garage $A 1 C 1$ has no collision and the vehicle critically pass. Another one is that vehicle point $D 1$ and the side of the garage $B D$ has no collision and the vehicle critically pass.The maximum value of the two critical values is the critical value of the system. As the figure shows, the two critical conditions are:

$$
\begin{gathered}
R_{C 1}=\sqrt{\left(R_{\min }-\frac{w}{2}\right)^{2}+c^{2}}=\sqrt{(l-c)^{2}+\left(R_{\min }-\frac{W_{1}}{2}\right)^{2}} \\
R_{D 1}=R_{\text {min }}+\frac{W_{2}}{2}=\sqrt{c^{2}+\left(R_{\text {min }}+\frac{w}{2}\right)^{2}}
\end{gathered}
$$

After calculating and simplifying:

$$
\begin{aligned}
& W_{1}=2 R_{\text {min }}-2 \sqrt{\left(R_{\text {min }}-\frac{w}{2}\right)^{2}+2 l c-c^{2}} \\
& W_{2}=2 \sqrt{\left(R_{\min }+\frac{w}{2}\right)^{2}+c^{2}}-2 R_{\text {min }} \\
& W_{\text {min }}=\max \left\{W_{1}, W_{2}\right\}
\end{aligned}
$$

For the depth of vertical parking space, as long as the length of the vehicle can be satisfied, that was: $H_{\text {min }}=l$

The above minimum parking space is the critical size of parking space. Actual garage needs to add a safe distance $\delta, \delta$ can be $(10 \sim 20 \mathrm{~cm})$. In the picture, the stopping spot in the process of reversing is coordinate origin. The two arc center coordinates of the trajectory are:

$$
\begin{aligned}
& O_{1}\left(R_{1_{-} \min } \sin \beta,-R_{1_{-} \min } \cos \beta\right) \\
& O_{2}\left(-R_{\text {min }} \cos \beta, R_{\text {min }} \sin \beta\right)
\end{aligned}
$$

By calculating, the distance from starting position of the two parking trajectory to point $B$ is:

$$
d^{\prime}=R_{1 \_ \text {min }} \sin \beta-R_{\min }(1-\cos \beta)-\frac{W}{2}
$$

According to the center coordinates, radius and rotation angle, equation of two paths are easy to get by diagram.

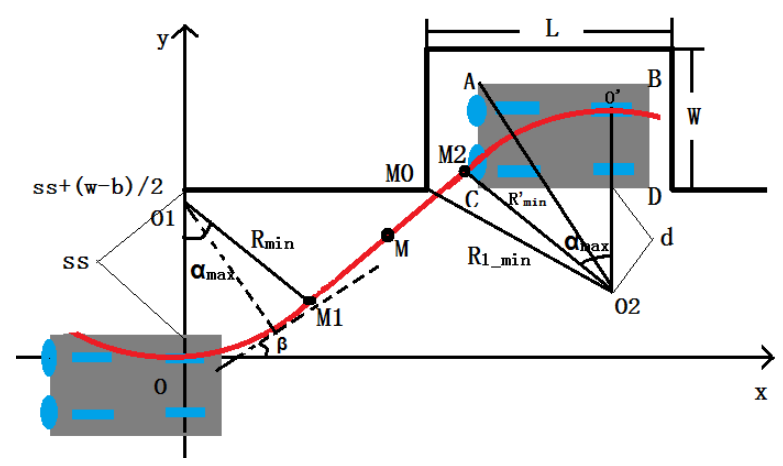

Fig. 2 Schematic diagram of automatic parallel parking path planning

The Fig. 2 showed the trajectory of automatic parallel parking, which had 3 stages: the arc-line-arc unsymmetrical path. $M 1, M 2$ were respectively tangent points of the two circular trajectory. The slope 
of the line was certain,meaning this path was also the asked minimum radius of trajectory when the maximum steering wheel turning angle was established. $\mathrm{L}$ and $\mathrm{W}$ were the effective length and width of the parking space respectively, ss was the distance between the left side of the garage and the right side of the vehicle, $R_{1_{\_} \min }$ was the trajectory radius of $\mathrm{A}, R_{2_{-} \min }$ was the trajectory radius of $\mathrm{B}$. It is likely to occur collisions between point $\mathrm{M} 0$ and $\mathrm{AC}$ side of the vehicle, also between the point $\mathrm{B}$ of the vehicle and the edge of garage inside.According the two possible collision locations, calculate the minimum efficient size of vehicle space. According to the turning radius of Ackerman angle, for circle O1:

$$
R_{\min }=\sqrt{R_{0 \_ \text {min }}^{2}-s^{2}}-b
$$

The turning radius of circle 02 was:

$$
R_{\text {min }}^{\prime}=\sqrt{R_{0 \_ \text {min }}^{2}-s^{2}}
$$

The critical conditions for the collisions of the above two places were:

$$
\begin{aligned}
& R_{1_{-} \min }=\sqrt{\left(R_{\text {min }}^{\prime}+\frac{w-b}{2}\right)^{2}+(s+a)^{2}}=\sqrt{\left(L_{\text {min }}-c\right)^{2}+\left(R_{\text {min }}^{\prime}-\frac{w+b}{2}\right)^{2}} \\
& R_{2_{-} \min }=\sqrt{\left(R_{\text {min }}^{\prime}+\frac{w-b}{2}\right)^{2}+c^{2}}=W_{\text {min }}-w+R_{\text {min }}^{\prime}+\frac{w-b}{2}
\end{aligned}
$$

Critical dimension of parking space was:

$$
\begin{aligned}
& L_{\text {min }}=c+\sqrt{\left(2 R_{\text {min }}^{\prime}-b\right) w+(s+a)^{2}} \\
& W_{\text {min }}=\sqrt{\left(R_{\text {min }}^{\prime}+\frac{w-b}{2}\right)^{2}+c^{2}}-R_{\text {min }}^{\prime}+\frac{w+b}{2}
\end{aligned}
$$

In the actual parking process, in order to prevent collisions, it need to add a certain safety distance $\delta, \delta$ was desirable $(10 \mathrm{~cm} \sim 20 \mathrm{~cm})$. In the trajectory given in this paper, it is assumed that the vehicle reversing into the parking space is just the end of the garage or has an interval safety distance.After the automatic parking,adjust the position of the vehicle manually, which means $\mathrm{O} 2$ is always in a straight line. The coordinates of several points in this trajectory are as follows:

$$
O_{1}\left(0, R_{\text {min }}\right) \quad, \quad M_{1}\left(R_{\text {min }} \sin \alpha_{\text {max }}, R_{\text {min }}\left(1-\cos \alpha_{\text {max }}\right)\right) \quad, \quad O_{2}\left(R_{\text {min }}^{\prime} \sin \alpha_{\text {max }}+x_{M_{2}}, s s+w-R_{\text {min }}^{\prime}\right),
$$

$M_{2}\left(\frac{y_{M_{2}}-y_{M_{1}}}{\tan \alpha_{\max }}+x_{M_{1}}, w+s s-R_{\text {min }}^{\prime}\left(1-\cos \alpha_{\text {max }}\right)\right)$. According to the coordinates of the key points, we can know the distance from the vehicle starting reversing position to the upper left corner of the parking space, that is $d^{\prime}=x_{\mathrm{O}_{2}}+c$ or $d^{\prime}=x_{\mathrm{O}_{2}}+c+\delta$. This distance is relevant to $s s$. Thus ss can be flexible to adjust the starting position of parking. Through the above coordinates, we can easily obtain the equation of the process of parking.

\section{Fuzzy control of vehicle speed}

In order to facilitate the control of the project of the vehicle reversing into garage, at the same time to adapt to different conditions, there was a need for keeping the vehicle speed at a constant speed, so the two dimensional fuzzy controller was needed to control the speed of the vehicle. With the speed error $E_{S}$ and the rate of the vehicle change $E C_{r}$ as the input, this paper chose the change of speed $u_{s}$ as output to dispel the speed error $[4,5]$. On the premise of meeting the requirements of vehicle trajectory control, as far as possible to reduce the numbers of linguistic variables and make the control rules simple and easy to operate, the input $E C_{r}$ and $u_{s}$ both select the value of the seven language variables $\{\mathrm{PB}, \mathrm{PM}, \mathrm{PS}, \mathrm{O}, \mathrm{NS}, \mathrm{NM}, \mathrm{NB}\}$. And in order to improve the steady-state accuracy, the input $E_{S}$ selected seven language variable values $\{\mathrm{PB}, \mathrm{PM}, \mathrm{PS}, \mathrm{O}, \mathrm{NS}, \mathrm{NM}, \mathrm{NB}\}$. The domain of $E_{S}$ and $E C_{r}$ is $\{-6,-5,-4,-3,-2,-1,0,1,2,3,4,5,6\}$. The domain of $\mathrm{u}$ is $\{-7,-6,-5,-4,-3,-2,-1,0,1,2,3,4$, $5,6,7\}$. That the domain of $u_{s}$ above selected 15 elements, in order to make the output more accurate. 
Fuzzy rules are the core of the fuzzy controller, which have a direct impact on the performance of the controller. Here, the fuzzy control rules can be designed according to the experience of the designers [6]. After the fuzzy sets and the domains of control of fuzzy variables error $E_{S}$, the error rate of change $E C_{r}$, change of speed $u_{s}$ were determined, the next step was to determine the membership functions of fuzzy linguistic variables, which are so-called fuzzy variables. If the actual error of sampling at the moment is $E_{S}$ and the variable rate of change is $E C_{r}$, according to the control rules the control result is:

$$
u_{s 1}=E_{S}\left[\left(N B_{E S}+N M_{E S}\right) \times P B u_{s}\right] \cdot E C_{r}\left[\left(N B_{E C r}+N M_{E C r}\right) \times P B u_{s}\right]
$$

For the $E_{S}$ and $E C_{r}$ membership function values corresponding to the level of the quantization to take 1 , and the rest are taken 0 values, so that the formula above can be simplified as:

$u_{s 1}=\min _{x}\left\{\max \left[\mu_{N B E S}(i), \mu_{N M E S}(i)\right], \max \left[\mu_{N B E C r}(j), \mu_{N M E C r}(j)\right], \mu_{P B u s}(x)\right\}$

where,$\mu_{N B E S}(i)$ and $\mu_{N M E S}(i)$ are the membership of the number of the $i$ element in the fuzzy set $N B_{E S}$ and $N M_{E S}, \mu_{N B E C r}(j)$ and $\mu_{N M E C r}(j)$ are the membership degree of the number of the $j$ element in the fuzzy set $N B_{E C r}$ and $N M_{E C r}$. The amount of control $u_{s 2} \ldots u_{s 21}$ can be determined by the rest control rules. The control amount is fuzzy set $u_{s}$, which is expressed as:

$$
u_{s}=u_{s 1+} u_{s 2+\ldots}+u_{s 21}
$$

Based on the results obtained, use the maximum membership method to change the control amount from fuzzy two variables to accurate amount. The main purpose of fuzzy control is to enable the vehicle to follow the reference trajectory, which can be adjusted in the deviation from the reference path, to ensure that the vehicle is always along the set track. This follow requires that the vehicle reference point is always in the vicinity of the reference path, and the direction of the vehicle is also consistent with the reference path tangent. By the above analysis, the input of the fuzzy controller was chosen as the deviation between the actual position and the reference position, including two basic errors. One is the position deviation between the actual vehicle reference point position and reference path $E P_{r}$, two is the angle deviation between the actual vehicle orientation angle and the reference path tangent angle $E G_{r}$. The path following of the vehicle is realized by controlling the vehicle steering. Thus the vehicle routing control system is a fuzzy control system with three inputs and one output structure. Although selecting many linguistic variables can make the control rules more comprehensive and more accurate, it also makes the control rules more complex. The computational complexity is greatly increased, and it will require higher capability. Involving the three variables for the automatic parking position, named the vehicle bias reference point to the horizontal and vertical coordinates and vehicle angle. In order to meet the requirements of vehicle routing control, minimizing the language variables' values to make the control rules easier, so the input variables $x, y$ and theta were all with five linguistic variables $\{\mathrm{PB}, \mathrm{PS}, \mathrm{O}, \mathrm{NS}, \mathrm{NB}\}$. At the same time, the output also selects five linguistic variables $\{\mathrm{PB}, \mathrm{PS}, \mathrm{O}, \mathrm{NS}, \mathrm{NB}\}$. Thus, in the case of a certain range of accuracy, the operating load of the controller is reduced as far as possible.

\section{Experiment and result analysis}

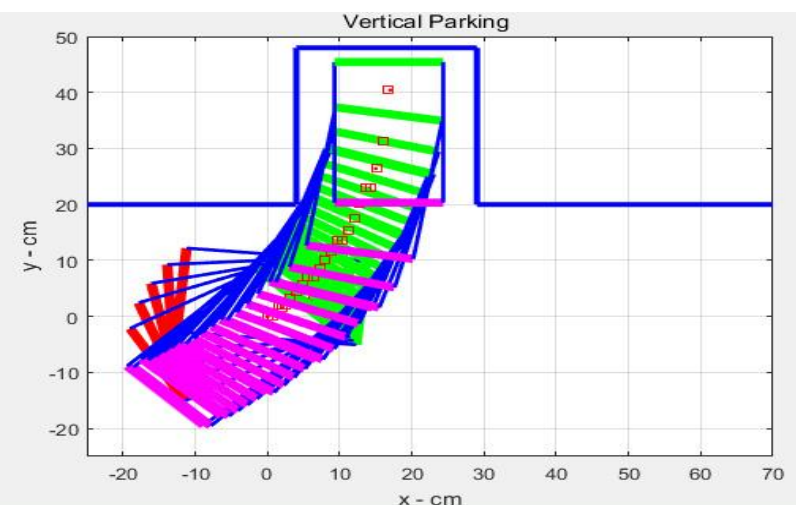

(a) Vertical parking parking simulation

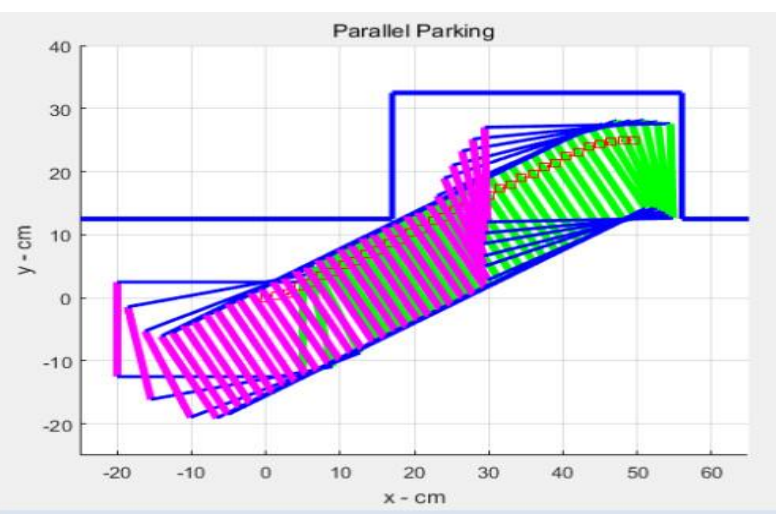

(b) parallel parking parking simulation

Fig. 3 A set of automatic vertical and parallel parking simulation 
In the Matlab Simulink simulation environment, after multiple sets of simulation, this paper verified the automatic parking system was reliable. Fig. 3 was one of the simulation results. Among them, vertical parking was divided into two stages, the forward phase (as shown in the red part on Fig. 3 (a)) and the backward phase (as shown in the purple part on Fig.3 (b)). The parameters values of the simulation were shown in Table 1.

Table 1. Parameters values of the simulation

\begin{tabular}{|c|c|c|c|c|c|c|c|c|}
\hline item & $\mathrm{L}[\mathrm{cm}]$ & $\mathrm{w}[\mathrm{cm}]$ & $\mathrm{s}[\mathrm{cm}]$ & $\mathrm{b}[\mathrm{cm}]$ & $\mathrm{c}[\mathrm{cm}]$ & $\mathrm{ss}[\mathrm{cm}]$ & $\alpha_{\max }$ & $\mathrm{v}[\mathrm{cm}]$ \\
\hline vertical parking & 25 & 15 & 10 & 10 & 5 & 15 & $\pi / 4$ & 3 \\
\hline parallel parking & 25 & 15 & 10 & 10 & 5 & 10 & $\pi / 2$ & 3 \\
\hline
\end{tabular}

\section{Conclusion}

The paper set up two parking schemes for two kinds of parking systems, using fuzzy control methods to control the position and speed of the vehicle, and to avoid the collisions. The system had the advantages of simple structure and good control effect, and achieved the goal of automatic parking. To make more accurate control, we must segment the input and output fuzzy variables, which leads to more complex fuzzy rules, having a great effect on the response speed of the micro controller.Therefore, in the control accurate and response speed, the system adopted a compromise control algorithm, which makes the response speed faster and the control precision higher.

\section{Acknowledgments}

This study of the paper was supported by natural science foundation of Jilin province (20170 101133JC), innovation and entrepreneurship training program for college students of Jilin University (2016B53477).

\section{References}

[1]Vorobieva H, Glaser S, Minoiu-Enache N, et al. Automatic parallel parking with geometric continuous-curvature path planning [C]. 2014 IEEE Intelligent Vehicles Symposium Proceedings. Ypsilanti, Michigan, USA. 8-11 June 2014,p.465-471.

[2]Gupta A, Divekar R, Agrawal M. Autonomous parallel parking system for Ackerman steering four wheelers[C].Computational Intelligence and Computing Research (ICCIC). Coimbatore India. 28-29 December 2010 ,p.1-6.

[3]Kim D, Chung W, Park S. Practical motion planning for car-parking control in narrow environment [J]. IET control theory \& applications, 2010, 4(1),p.129-139.

[4]Kwon H, Chung W. Performance analysis of path planners for car-like vehicles toward automatic parking control[J]. Intelligent Service Robotics, 2014, 7(1),p.15-23.

[5]Sungwoo C, Boussard C, d'Andréa-Novel B. Easy path planning and robust control for automatic parallel parking[J]. IFAC Proceedings Volumes, 2011, 44(1),p. 656-661.

[6]Vorobieva H, Minoiu-Enache N, Glaser S, et al. Geometric continuous-curvature path planning for automatic parallel parking[C]. Networking, Sensing and Control (ICNSC).Evry, France.10-12 April 2013, p.418-423. 